\title{
Ductal adenocarcinoma of the prostate: immunohistochemical findings and clinical significance
}

This article was published in the following Dove Press journal:

OncoTargets and Therapy

23 October 2013

Number of times this article has been viewed

Jianjun Sha',2
Juanjie Bo'
Jiahua Pan'
Lianhua Zhang'
Hanqing Xuan'
Wei Chen'
Dong Li'
Zhaoliang Wang'
Dongming Liu'
Yiran Huang
'Department of Urology, Renji
Hospital, School of Medicine, Shanghai
Jiaotong University, Shanghai, 'School
of Biomedical Engineering, Shanghai
jiaotong University, Shanghai, People's
Republic of China

Correspondence: Yiran Huang Department of Urology, Renji Hospital, Shanghai Jiaotong University School of Medicine, Shanghai 2000I, People's Republic of China

Tel +86 2l 53882029

Fax +86 2I 68383543

Email yiranhuangI@I26.com
Introduction: To investigate the clinical features, diagnosis, treatment, and prognosis of ductal adenocarcinoma of the prostate.

Methods: The clinicopathological and immunohistochemical data of seven patients with ductal adenocarcinoma of the prostate were retrospectively analyzed. All patients underwent physical examination, magnetic resonance imaging (MRI), bone scan, cystoscopy, and computed tomography (CT) scan. The level of prostate-specific antigen (PSA) before and after surgery was assessed. Different prostate cancer markers were used for immunohistochemical staining.

Results: The mean age of the seven patients diagnosed with prostatic ductal adenocarcinoma in this study was 76.2 years (range 57-88). Five patients presented with intermittent and painless gross hematuria, one patient with progressive dysuria, and one patient with elevated serum PSA on routine health examination. The level of PSA before surgery ranged from 1.3 to $45.0 \mathrm{ng} / \mathrm{mL}$. Immunohistochemical staining results of the prostatic ductal adenocarcinoma confirmed positivity for PSA, prostatic acid phosphatase, androgen receptor, and alpha-methyacyl co-enzyme A (CoA)-reductase markers. Two of the patients underwent bilateral orchiectomy combined with anti-androgen therapy, three underwent transurethral resection of prostate, one received radical prostatectomy, and one received medical castration therapy. The clinical outcomes of all patients were satisfactory, based on follow-up data. The symptoms of hematuria and dysuria were ameliorated well, and the postoperative PSA level decreased below $4.0 \mathrm{ng} / \mathrm{mL}$. Recurrence or metastasis of disease was not detected on MRI and bone scan.

Conclusion: Ductal adenocarcinoma of the prostate is a rare subtype of prostate carcinoma, the diagnosis of which could be based on pathological and immunohistochemical examination. Earlier management promises better prognosis.

Keywords: ductal adenocarcinoma, immunohistochemistry, pathology, prognosis, prostate

\section{Introduction}

Ductal adenocarcinoma of the prostate is a rare histologic subtype of prostate carcinoma. The incidence of ductal adenocarcinoma is less than $1 \%$ and it mainly affects elderly men. ${ }^{1,2}$ Ductal adenocarcinoma is characterized by gross hematuria or urinary obstructive symptoms. Prostatic ductal carcinoma (PDC) mainly arises from large primary peri-urethral prostatic ducts. However, tumors arising from other peri-urethral prostatic ducts are known as acinar prostate carcinoma. In many instances, ductal adenocarcinoma is found in association with the more common acinar carcinoma. ${ }^{3}$

The diagnosis of PDC generally depends on histopathologic and immunohistochemical examination. The most common patterns seen in PDC are papillary and cribriform. The papillary formation pattern distinguishes between ductal and acinar 
adenocarcinoma, as it is absent in acinar adenocarcinoma of the prostate. ${ }^{3}$ Ductal adenocarcinoma of the prostate mainly expresses immunohistochemical markers of prostatic tissue, including prostate-specific antigen (PSA), prostatic acid phosphatase (PAP), alpha-methylacyl coenzyme A (CoA)-reductase (AMACR), androgen receptor (AR), and cytokeratin (CK) $7 .^{4}$ The immunohistochemical evaluation of ductal adenocarcinoma is markedly improved by using basal cell markers known as "negative markers". Basal cells are detected using proto-oncogene (p63), high-molecular-weight CK, 34ßE12, and CK5/6.

Expression of immunohistochemical markers plays an important role as a prognostic indicator in PDC of the prostate. This retrospective study was conducted to assess the clinical manifestations, diagnostic findings, treatment, and prognosis of patients with ductal adenocarcinoma of the prostate in the People's Republic of China.

\section{Materials and methods \\ Patients}

Patient enrollment criteria for this study were as follows: patients were newly diagnosed with prostatic ductal adenocarcinoma, patients were untreated, and were without a history of other types of tumors. Seven patients were identified from pathology records of patients admitted to the Renji Hospital, Shanghai Jiaotong University School of Medicine, People's Republic of China, and reviewed retrospectively. Specimens were obtained after laparoscopic radical prostatectomy (one case), at transurethral resection of the prostate (TURP) (three cases), and after prostate biopsy (three cases). Data were collected from January 2005 to December 2010.

\section{Immunohistochemical analysis}

All tissue samples were fixed in 10\% neutral formaldehyde solution, conventionally dehydrated, and embedded in paraffin. Sections of 3-4 $\mu \mathrm{m}$ were sliced, dipped out, and dried. These slices were then baked for $2-3$ hours at $56^{\circ} \mathrm{C}$ in an oven. Sections were then dewaxed using xylene and alcohol and hydrated with distilled water. After incubation with $3 \%$ aqueous $\mathrm{H}_{2} \mathrm{O}_{2}$ for 10 minutes to block endogenous peroxidase, antigen retrieval was carried out by the microwave high-temperature method. The slides were then incubated with the primary antibody (Table 1 ) at $37^{\circ} \mathrm{C}$ for 1 hour and washed three times with tri-buffered saline (TBS). Afterwards, the slides were incubated with the secondary antibody (rabbit/mouse anti-human monoclonal antibody) at $37^{\circ} \mathrm{C}$ for 30 minutes to 1 hour and washed three times with TBS. For developing enzyme activity, diaminobenzidine
Table I List of primary antibodies used in immunohistochemical staining

\begin{tabular}{lll}
\hline Antibody & Dilution & Source \\
\hline PAP & I:200 & Changdao \\
PSA & $1: 50$ & Gene \\
$34 \beta E I 2$ & $1: 50$ & Gene \\
P63 & Working solution & Changdao \\
AMACR & Working solution & Maixin \\
Ki67 & I:50 & Changdao \\
P53 & $1: 50$ & Gene \\
AR & Working solution & Gene \\
CK 5/6 & Working solution & Gene \\
\hline
\end{tabular}

Abbreviations: AMACR, alpha-methylacyl coenzyme A (CoA)-reductase; AR, androgen receptor; CK, cytokeratin; PAP, prostatic acid phosphatase; PSA, prostatespecific antigen.

(DAB) staining was conducted for 5-10 minutes at room temperature. The sections were counterstained with hematoxylin for 10-60 seconds, dried at room temperature, cleared in xylene, and mounted with neutral resin under the microscope. Immunohistochemistry slides purchased from EnVision Peripherals, Inc. (Fremont, CA, USA) were used as positive controls, while tissue slides incubated with TBS buffer instead of primary antibodies served as negative controls. Immunohistochemical analysis was conducted to detect the expression of cancer markers such as serum PSA, PAP, AR, and AMACR, and basal cell markers such as $34 \beta E 12$, p63, and CK5/6.

\section{Clinical evaluation}

Patients underwent a digital rectal examination (DRE) to detect the size of the enlarged prostate gland. Magnetic resonance imaging (MRI) of the prostate, and bone scan, were conducted to evaluate the stage of prostate cancer. Transrectal ultrasonography, chest and abdomen computed tomography (CT), and cystoscopy were also performed. The prognosis of mixed types of prostate adenocarcinoma was assessed using the Gleason score, based on the appearance of the carcinoma and its spreading pattern. The Gleason score has not been demonstrated to be useful in predicting prognosis for prostate ductal adenocarcinoma.

\section{Treatment}

The main therapy for PDC consists of surgery, radiotherapy, and anti-androgen therapy. Patients with prostate ductal adenocarcinoma received TURP. However, patients with elevated serum PSA underwent prostate biopsy. Patients who were pathohistologically confirmed with prostate ductal adenocarcinoma underwent radical prostatectomy. Patients also received medical castration plus anti-androgen treatment 
with $3.6 \mathrm{mg}$ goserelin acetate $\left(\right.$ Zoladex $^{\circledR}$, Astra Zeneca, Ltd., London, UK) administered subcutaneously once every 28 days plus oral bicalutamide amine $50 \mathrm{mg}$ daily.

\section{Follow-up}

Regular outpatient follow-up was conducted for each patient to determine the progression of disease. Patients were assessed for change in PSA level. MRI, bone scan, and Gleason scores were used to assess disease progression. The patients were followed-up every 3 months for the initial 2 years, and then every 6 months.

\section{Results}

\section{Clinical data}

Clinical data for seven patients with ductal adenocarcinoma of the prostate, with a mean age of 76.2 years (range 57-88), are shown in Table 2. Of the seven patients, five presented with intermittent and painless gross hematuria for a period ranging from 2 weeks to 3 months, one presented with progressive dysuria for about 3 years, and one presented with elevated serum PSA from a routine health examination (Table 2). Only two patients were found to have hard nodules in the prostate DRE, while no other patients showed any abnormalities. The PSA level of the patients ranged from 1.3 to $45.0 \mathrm{ng} / \mathrm{mL}$ before surgery, including four patients with normal PSA levels $(<4.0 \mathrm{ng} / \mathrm{mL})$, one patient with a value of $9.2 \mathrm{ng} / \mathrm{mL}$, and the other two patients with a level of more than $10 \mathrm{ng} / \mathrm{mL}$. Postoperative PSA levels were in the normal range (0.03-3.78 ng/mL). Preoperative whole-body radionuclide bone scans revealed that two patients had bone metastases. T2-weighted MRI images of the prostate showed that there were low-intensity lesions at both the peripheral region and the extracapsular extension of the right peripheral region. Postoperative bone scan, transrectal ultrasound, and prostate MRI showed no recurrence of prostate tumors, or locally advanced or metastatic disease. Prognosis was good for all patients, except for one patient who died of myocardial infarction.

\section{Histology}

The pathological examination of tumors from all seven patients showed papillary and tubular structures, high columnar epithelium, single or pseudo-stratified arrangements of the nucleus inside the basement membrane, and prominent cell atypia, which were similar to well differentiated endometrial cancer (Figures 1-3). Histological analysis showed that the tumors from three patients were pure prostatic ductal adenocarcinoma, and the remaining

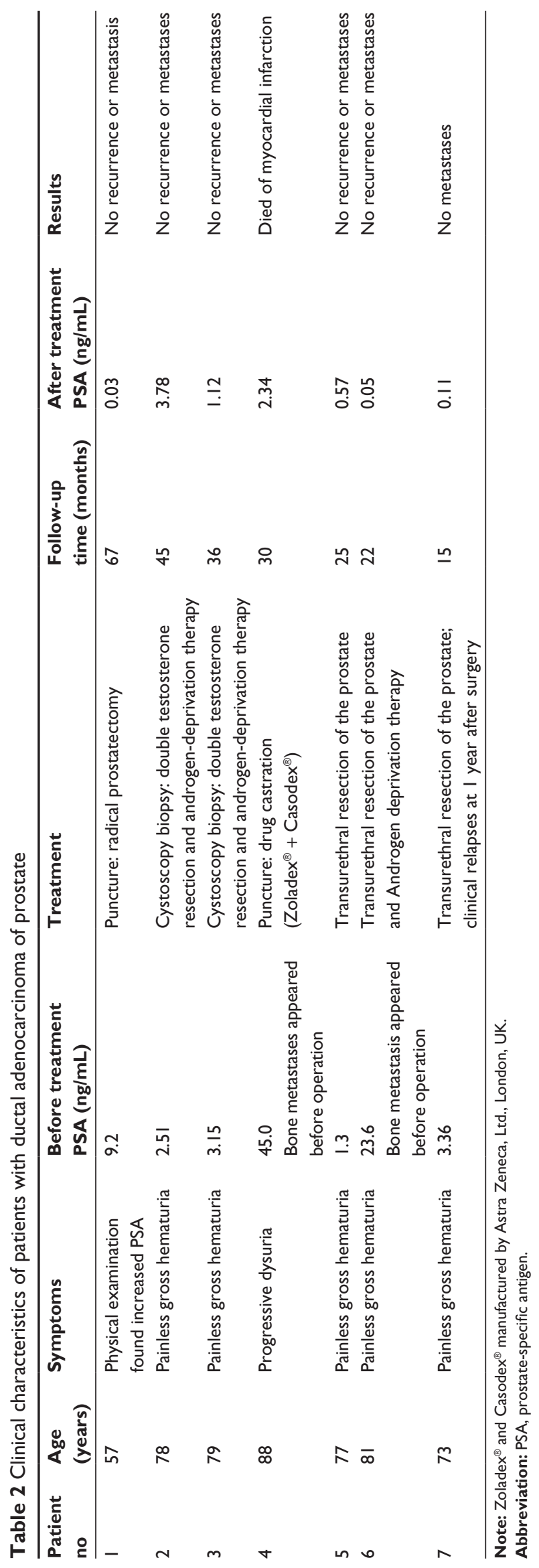



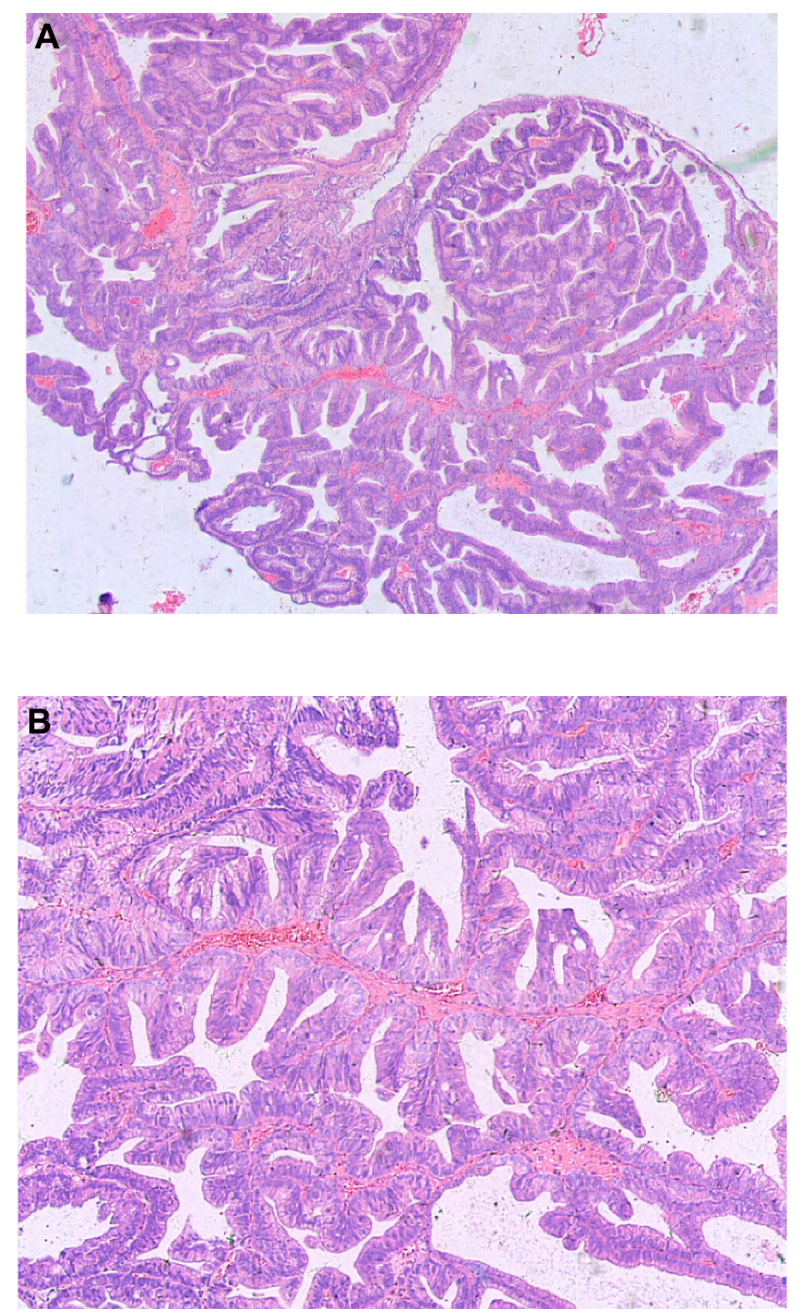

Figure I Prostatic ductal adenocarcinoma tumor cells showing a papillary histological pattern.

Note: A, hematoxylin and eosin $\times 25$; B hematoxylin and eosin $\times 50$.

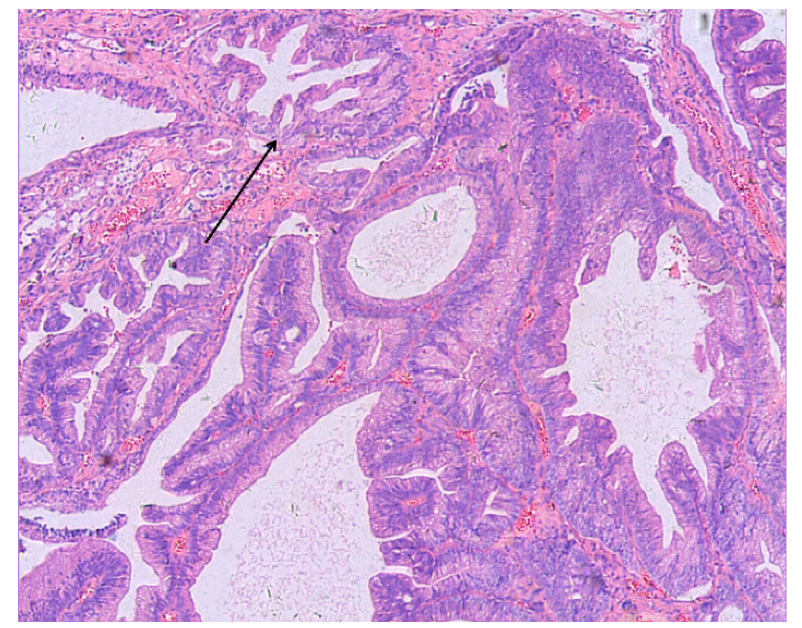

Figure 2 Prostatic ductal adenocarcinoma tumor made of back-to-back glands, which have a bridge-like structure within the glands. The glandular cavity was fissure type (see arrow). Hematoxylin and eosin $\times 50$.

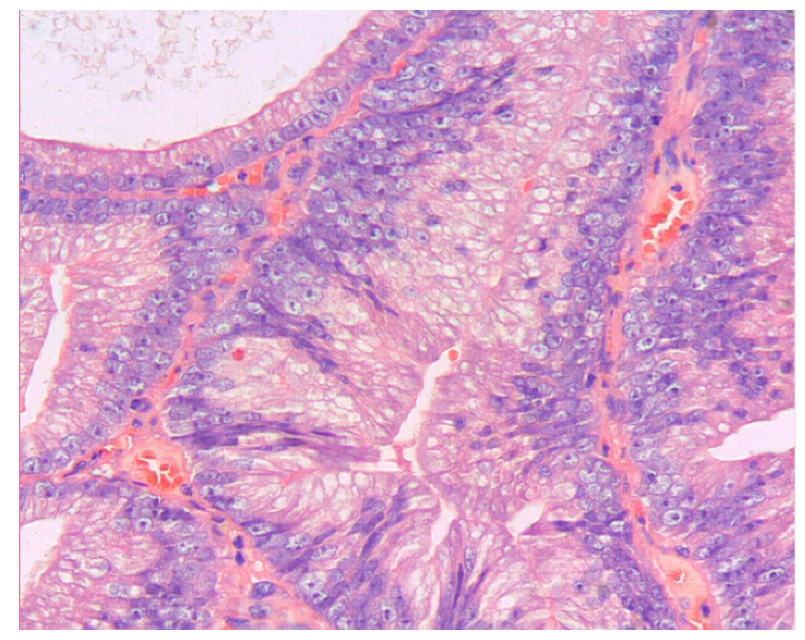

Figure 3 Prostatic ductal adenocarcinoma tumor cells are high columnar and amphophil, surrounded by abundant cytoplasm. Tumor cells are arranged in single or pseudostratified layers. Hematoxylin and eosin $\times 200$.

four demonstrated a mixed histology pattern, with both prostate ductal adenocarcinoma and the classic small acinar prostate adenocarcinoma. The conventional acinar Gleason score was reported for the four mixed-type patients. Mixed classic adenocarcinoma was found mostly in patients with high Gleason scores, with one score of seven, two scores of eight, and one score of nine.

\section{Immunohistochemistry}

The immunohistochemical analysis showed that all patients were positive for cancer markers, including PSA, PAP, AR, and AMACR. However, six of the seven patients were negative for the basal cell markers 34ßE12, p63, and CK5/6, and one patient tested slightly positive for these basal cell markers.

\section{Treatment and follow-up}

Patient follow-up periods ranged from 15 to 67 months in this study. Cystoscopy examination revealed cauliflowershaped tumors in the prostatic urethra of all patients, which were morphologically similar to the prostatic ductal adenocarcinoma in two of the seven patients. These two patients underwent biopsy, and prostatic ductal adenocarcinoma was subsequently confirmed, before receiving dual orchiectomy and anti-androgen therapy. Three patients underwent TURP to remove the cauliflower-shaped tumor and the base of the prostate tissue. Subsequent postoperative pathological examination identified prostatic ductal adenocarcinoma. Two patients with elevated serum PSA levels underwent prostate biopsy that identified prostate ductal adenocarcinoma; one of the two patients received radical prostatectomy and the other 
received medical castration plus anti-androgen therapy. Of the seven patients, six did not report any gross hematuria, dysuria, bone pain, and other symptoms, while one patient who underwent TURP presented with painless gross hematuria 1 year after resection and received TURP again. Pathology features remained the same as the PDC.

\section{Discussion}

Prostate cancer is the second most commonly diagnosed cancer worldwide, with a relatively high mortality rate. This disease mainly affects elderly men. ${ }^{6}$ Prostate cancer is more prevalent in developed countries, such as the USA. Although the incidence and mortality rate of prostate cancer is lower in Asian countries, the incidence is on the rise. ${ }^{7}$ The estimated incidence of prostate cancer in the People's Republic of China is $2.1 \%$, with a mortality rate of $1.2 \%{ }^{6}$

The first incidence of ductal adenocarcinoma, a rare variant of prostate cancer that did not demonstrate the typical characteristics of prostate adenocarcinoma in the early stages, such as elevated PSA and palpation induration, was reported by Melicow and Patcher in $1967 .{ }^{3}$ The atypical presentation of prostatic ductal adenocarcinoma meant the condition remained unnoticed and therefore untreated. Prostatic ductal adenocarcinoma was originally termed "endometrioid carcinoma" due to its histologic resemblance to the uterine endometrial carcinoma, and it is suspected that the tumor arises from the prostatic utricle, which is the male homologue of the female uterus. Recently, it was renamed to "ductal carcinoma with endometrioid features" and then to "prostatic ductal adenocarcinoma". ${ }^{3}$ A diagnosis of ductal adenocarcinoma may be difficult, because, instead of showing typical clinopathological features of the acinar type of prostatic cancer, ductal adenocarcinoma closely resembles the uterine endometrioid carcinoma developed from the prostatic utricle and other primary or locally invasive tumors arising from the urothelial tract. In addition, the frequent coexistence of prostatic ductal and acinar adenocarcinoma makes diagnosis of ductal adenocarcinoma difficult. With advances in molecular diagnostic tools, it may become possible to differentiate prostatic ductal adenocarcinoma from other types of prostatic malignancy based on molecular biomarkers.

All patients assessed in our study were elderly males, five of whom presented with hematuria as the first clinical symptom. Three cases were confirmed to be the single histology type of prostatic ductal adenocarcinoma based on pathological analysis. The diagnosis of prostatic ductal adenocarcinoma relies mainly on pathological and immunohistochemical examination, which could be used to distinguish prostatic ductal adenocarcinoma from other types of prostate neoplasm, such as the classic adenocarcinoma of the prostate, high-grade prostatic intra-epithelial neoplasia, primary prostate transitional cell carcinoma, and metastatic adenocarcinoma. ${ }^{8}$ Traditionally, fissure type glandular cavities and glands with bridge-like structures can be seen in prostatic ductal adenocarcinoma (Figure 2). The tumor cells of prostatic ductal adenocarcinoma are high columnar, amphophil, and arranged in single or pseudostratified layers (Figure 3). In addition, prostatic ductal adenocarcinoma shows positive immunostaining for PSA, PAP, and AR, while transitional cell carcinoma is negative. A combination of tumor markers, such as AMACR, PSA, and PAP, are used to identify tumors that are derived from the prostate epithelium, while a combination of $34 \beta \mathrm{E} 12$ and p63 (markers of basal cells of prostate) are helpful to identify tumors arising from basal cells. Researchers deemed that high-molecular-weight CK proteins (HMWCK, 34ßE12, CK20) and p63 cocktails aid in the diagnosis of prostate basal cells. ${ }^{4,9,10}$ In this study, $43 \%$ of patients $(3 / 7)$ had pure type ductal adenocarcinoma, and the remaining patients (4/7) were associated with classic adenocarcinoma of small acinar structure. All patients in our study were confirmed positive for PAP, AMACR, and AR.

Low levels of PSA have been seen in prostate ductal adenocarcinoma derived from the prostatic ductal epithelial cells, while PSA level rises when the tumor begins to progress and invade the surrounding glandular tissue. ${ }^{11}$ Therefore, the possibility of prostatic ductal adenocarcinoma should still be considered when patients present without prostate nodules or an elevated PSA. The PSA levels of four patients in our study were within the normal range before treatment, which indicates that these patients may be in early clinical stages of the disease.

Treatment modalities for prostatic ductal adenocarcinoma includes surgery (radical prostatectomy, TURP), endocrine therapy, and radiation therapy (brachytherapy and external radiation therapy), or combined treatments. It is noteworthy that the prostatic ductal adenocarcinoma is sensitive to endocrine therapy and radiotherapy, which have been used for patients with advanced prostatic ductal adenocarcinoma. ${ }^{12}$ Eade et $\mathrm{al}^{12}$ reported that the level of PSA declined when prostate cancer patients received radiotherapy, and all six cases of prostatic ductal adenocarcinoma showed no local tumor recurrence, along with a significantly prolonged survival time. Huang et $\mathrm{al}^{13}$ reported that prostate ductal adenocarcinoma was sensitive to endocrine therapy, which was consistent with our study findings that endocrine therapy 
was effective in all three patients who received anti-androgen therapy.

There are conflicting results in the literature regarding the prognosis of prostatic ductal adenocarcinoma. Brinker et $\mathrm{al}^{14}$ suggested that prostatic ductal adenocarcinoma was associated with a poorer prognosis than the acinar type of prostate cancer. Christensen et $\mathrm{l}^{15}$ found a higher recurrence rate with prostate ductal adenocarcinoma than acinar carcinoma in patients receiving radical prostatectomy. However, Aydin et al $^{16}$ stated that prostatic ductal adenocarcinoma was less malignant, slower to progress, and carried a better prognosis than the typical acinar prostate cancer. Tu et $\mathrm{al}^{17}$ reported that prognosis was comparable to other common types of prostate cancer in 25 cases of single histological type prostatic ductal adenocarcinoma, with an overall survival of 13.8 years. Some researchers believe that prognosis for prostatic ductal adenocarcinoma can be closely related to the clinical stage, tumor location, depth of invasion, and other prostate diseases. ${ }^{16,18,19}$ Prognosis of patients receiving different treatments were all excellent in our study, probably due to the low clinical staging and early diagnosis and treatment. However, this conclusion needs to be further investigated in a large clinical trial.

In summary, our study suggests that diagnosis of ductal adenocarcinoma of the prostate can mainly be based on pathological and immunohistochemical examinations. Early detection of the disease and early treatment may lead to a better prognosis. TURP may be an effective treatment strategy that can be used in the treatment of both pure and mixed types of prostate ductal adenocarcinoma.

\section{Acknowledgment}

This study was supported by National Natural Science Foundation of the People's Republic of China (91129725).

\section{Disclosure}

The authors report no conflicts of interest in this work.

\section{References}

1. Bostwick DG, Kindrachuk RW, Rouse RV. Prostatic adenocarcinoma with endometrioid features. Clinical, pathologic, and ultrastructural findings. Am J Surg Pathol. 1985;9:595-609.

OncoTargets and Therapy

\section{Publish your work in this journal}

OncoTargets and Therapy is an international, peer-reviewed, open access journal focusing on the pathological basis of all cancers, potential targets for therapy and treatment protocols employed to improve the management of cancer patients. The journal also focuses on the impact of management programs and new therapeutic agents and protocols on
2. Gong Y, Caraway N, Stewart J, Staerkel G. Metastatic ductal adenocarcinoma of the prostate: cytologic features and clinical findings. $\mathrm{Am} \mathrm{J}$ Clin Pathol. 2006;126:302-309.

3. Melicow MM, Patcher MR. Endometrial carcinoma of prostatic utricle (uterus masculinus). Cancer. 1967;20:1715-1722.

4. Herawi M, Epstein JI. Immunohistochemical antibody cocktail staining (p63/HNWCK/AMACR) of ductal adenocarcinoma and Gleason pattern 4 cribriform and noncribiform acinar adenocarcinomas of the prostate. Am J Surg Pathol. 2007;31:889-894.

5. Shah RB, Zhou M, LeBlanc M, et al. Comparison of the basal cellspecific markers, 34betaE12 and p63, in the diagnosis of prostate cancer. Am J Surg Pathol. 2002;26:1161-1168.

6. Ferlay J, Shin HR, Bray F, Forman D, Mathers C, Parkin DM. Estimates of worldwide burden of cancer in 2008: GLOBOCAN 2008. Int $J$ Cancer. 2010;127:2893-2917.

7. Baade PD, Youlden DR, Krnjacki LJ. International epidemiology of prostate cancer: geographical distribution and secular trends. Mol Nutr Food Res. 2009;53:171-184.

8. Greene LF, Farrow GM, Ravits JM, Tomera FM. Prostatic adenocarcinoma of ductal origin. J Urol. 1979;121:303-305.

9. Chen JQ, Jiang ZM, Zhang HZ, et al. The diagnostic value of immunohistochemistry in the prostate biopsy. J Diagnostic Pathol. 2002;9:70-72.

10. Zhou M, Shah R, Shen R, Rubin MA. Basal cell cocktail (34betaE12 + p63) improves the detection of prostate basal cells. Am J Surg Pathol. 2003;27:365-371.

11. Oxley JD, Abbott CD, Gillatt DA, MacIver AG. Ductal carcinomas of the prostate: a clinicopathological and immunohistochemical study. Br J Urol. 1998;81:109-115.

12. Eade TN, Al-Saleem T, Horwitz EM, Buyyounouski MK, Chen DY, Pollack A. Role of radiotherapy in ductal (endometrioid) carcinoma of the prostate. Cancer. 2007;109:2011-2115.

13. Huang SS, Wu DL, Zhang HZ, et al. Analysis of clinical pathology, and treatment characteristics of the prostate ductal adenocarcinoma. Chin J Urol. 2009;30:382-385. Chinese.

14. Brinker DA, Potter SR, Epstein JI. Ductal adenocarcinoma of the prostate diagnosed on needle biopsy: correlation with clinical and radical prostatectomy findings and progression. Am J Surg Pathol. 1999;23:1471-1479.

15. Christensen WN, Steinberg G, Walsh PC, Epstein JI. Prostatic duct adenocarcinoma. Findings at radical prostatectomy. Cancer. 1991;67: 2118-2124.

16. Aydin H, Zhang J, Samaratunga H, et al. Ductal adenocarcinoma of the prostate diagnosed on transurethral biopsy or resection is not always indicative of aggressive disease: implications for clinical management. BJU Int. 2010;105:476-480.

17. Tu SM, Lopez A, Leibovici D, et al. Ductal adenocarcinoma of the prostate: clinical features and implications after local therapy. Cancer. 2009;115:2872-2880.

18. Elgamal AA, Van de Voorde W, Van Poppel H, Vandeursen H, Baert L, Lauweryns J. Exophytic papillary prostatic duct adenocarcinoma with endometrioid features, occurring in prostatic urethra after TURP. Urology. 1994;43:737-742.

19. He JY, Wang WZ, Shi GW, et al. Ductal adenocarcinoma of the prostate (a case report and literature review). Chin J Urol. 2000;21:177. Chinese.

\section{Dovepress}

patient perspectives such as quality of life, adherence and satisfaction. The manuscript management system is completely online and includes a very quick and fair peer-review system, which is all easy to use. Visit http://www.dovepress.com/testimonials.php to read real quotes from published authors. 